\section{Case Reports in Neurology}

Case Rep Neurol 2020;12:447-451

DOI: $10.1159 / 000510395$

Published online: November 17, 2020
This article is licensed under the Creative Commons Attribution-NonCommercial 4.0 International License (CC BY-NC) (http://www.karger.com/Services/OpenAccessLicense). Usage and distribution for commercial purposes requires written permission.

\title{
Prominent Prolongation of Cortical Silent Period Induced by Transcranial Magnetic Stimulation in Creutzfeldt-Jakob Disease
}

\author{
Hideyuki Matsumoto ${ }^{a}$ Naohiro Uchio ${ }^{a}$ Akihito $\mathrm{Hao}^{\mathrm{a}}$ Mari Haga ${ }^{\mathrm{b}}$ \\ Chiaki Abe $^{\mathrm{b}}$ Yuuri Sakamoto $^{\mathrm{b}}$ Yoshikazu Ugawa $^{\mathrm{c}}$ \\ aDepartment of Neurology, Mitsui Memorial Hospital, Tokyo, Japan; bepartment of \\ Clinical Laboratory, Mitsui Memorial Hospital, Tokyo, Japan; 'Department of Human \\ Neurophysiology, School of Medicine, Fukushima Medical University, Fukushima, Japan
}

\section{Keywords}

Transcranial magnetic stimulation · Motor-evoked potential · Creutzfeldt-Jakob disease · Cortical silent period $\cdot$ Periodic synchronous discharge $\cdot$ Periodic myoclonus

\begin{abstract}
The cortical silent period (CSP) induced by transcranial magnetic stimulation (TMS) has been reported to be prolonged in 2 Creutzfeldt-Jakob disease (CJD) patients who presented with periodic myoclonus. Herein, we will show a prominent prolongation of TMSinduced CSP in a patient with CJD who did not have periodic myoclonus. The patient was a 66-year-old woman who developed rapidly progressive dementia. No myoclonic jerks were observed. Brain magnetic resonance imaging showed high-intensity lesions in the cerebral cortex, basal ganglia, and thalamus on diffusion-weighted images. Electroencephalography (EEG) showed diffuse and continuous slow waves, but no periodic synchronous discharges (PSDs). A TMS study revealed that the duration of CSP was prominently prolonged: the duration of CSP $(370 \mathrm{~ms})$ equaled that of the mean +6.5 SD of the normal value. One month after admission, the patient exhibited akinetic mutism and developed periodic myoclonus in her limbs. The clinical course was compatible with CJD. To date, CSP has been measured in only 2 CJD patients. The common findings in both cases were
\end{abstract}




\section{Case Reports in Neurology}

Case Rep Neurol 2020;12:447-451

DOI: 10.1159/000510395

(c) 2020 The Author(s). Published by S. Karger AG, Basel www.karger.com/crn

Matsumoto et al.: Prominent Prolongation of Cortical Silent Period Induced by Transcranial Magnetic Stimulation in CJD

marked prolongation of CSP, periodic myoclonus, and PSD on EEG. In short, we demonstrated that TMS-induced CSP was prominently prolonged even at the early stage of CJD without periodic myoclonus or PSD. In other disorders, the CSP has not been reported to be comparably prolonged to that of CJD patients. Therefore, we conclude that TMS-induced CSP could be prominently prolonged even in the early stage of CJD. The marked prolongation of the CSP might be an early biomarker of CJD.

(C) 2020 The Author(s)

Published by S. Karger AG, Basel

\section{Introduction}

Creutzfeldt-Jakob disease (CJD) is a prion disease caused by the conversion of the normal prion protein (normal cellular form of prion protein; PrPC) into an abnormal form of the protein (scrapie form of prion protein; PrPSc). The deposition of PrPSc in the brain causes degenerative encephalopathy with rapidly progressive dementia. To make a diagnosis of CJD, other diseases, such as autoimmune encephalopathy, should be differentiated. A few papers reported findings of transcranial magnetic stimulation (TMS) in CJD [1]. The cortical silent period (CSP) induced by TMS right after a motor-evoked potential (MEP) has been reported to be prolonged in 2 CJD patients who presented with periodic myoclonus [2, 3]. In the present article, we will show a prominent prolongation of TMS-induced CSP in a patient with CJD who did not have periodic myoclonus.

\section{Case Report}

The patient was a 66-year-old woman who noticed gait instability and slowness of speech 2 months prior to admission. Her symptoms deteriorated rapidly, and she developed visual hallucinations, difficulty in writing and reading, and insomnia 1 month prior to admission. She was unable to walk without support and was admitted to our hospital. She had no remarkable past, social, or family histories.

Neurological examinations revealed cognitive impairment (Mini-Mental State Examination 20/30), including disorientation, and impairment of memory, calculation, reading, and writing. She had nonfluent speech and poor comprehension. In all extremities, hypertonus, normal tendon reflexes, and flexor planter responses were noted. No myoclonic jerks were observed. Brain magnetic resonance imaging (MRI) showed high-intensity lesions in the cerebral cortex, basal ganglia, and thalamus on diffusion-weighted images and T2-weighted images (Fig. 1a). Cerebrospinal fluid examinations showed normal cell count, total protein, and positive 14-3-3 protein. Electroencephalography (EEG) showed diffuse and continuous slow waves such as $\theta$ waves and $\delta$ waves, but no periodic synchronous discharges (PSDs) (Fig. 1b). Surface electromyograms (EMGs) showed irregular positive multifocal myoclonus and irregular negative myoclonus (Fig. 1c). Neither giant somatosensory-evoked potential, exaggerated C-reflex, nor cortical reflex negative myoclonus was elicited by electrical stimulation of the median nerve.

TMS was applied to the primary motor cortex using the same method as that reported previously [4]. The following parameters were measured: active motor threshold (AMT), resting motor threshold (RMT), central motor conduction time (CMCT), and CSP. MEPs were recorded from the first dorsal interosseous muscle (FDI). To measure CSP, stimulus intensity was set at RMT $120 \%$ or RMT $140 \%$. At each stimulus intensity, CSP was measured 8 times during 


\section{Case Reports in Neurology}

Case Rep Neurol 2020;12:447-451

DOI: 10.1159/000510395

(c) 2020 The Author(s). Published by S. Karger AG, Basel www.karger.com/crn

Matsumoto et al.: Prominent Prolongation of Cortical Silent Period Induced by Transcranial Magnetic Stimulation in CJD

voluntary contraction. The duration of CSP was measured from the onset of MEP to the end of CSP. The average CSP duration from 8 single recordings was used as the representative data of CSP duration. All MEP parameters were compared with normal values obtained from 28 age-matched, normal subjects.

The AMT was 30\% for the right FDI and 30\% for the left FDI (normal values: $35.2 \pm 6.3 \%$ [mean \pm SD]). The RMT was 36 and $34 \%$ for the right and left FDI, respectively (normal values: $50.6 \pm 9.0 \%$ ). The CMCTs were $7.0 \mathrm{~ms}$ for both FDIs (normal values: $7.0 \pm 0.4 \mathrm{~ms}$ ). The duration

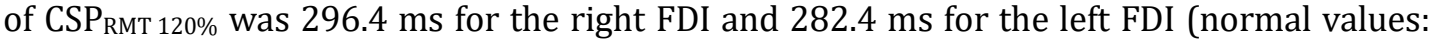
$107.1 \pm 35.2 \mathrm{~ms}$ ). The CSP RMT $140 \%$ was 365.1 and $382.4 \mathrm{~ms}$ for the right and left FDI, respectively (normal values: $134.5 \pm 36.1 \mathrm{~ms}$ ).

One month after admission, the patient exhibited akinetic mutism and developed periodic myoclonus in her limbs. The EEG-EMG polygraph showed periodic positive-negative myoclonus associated with PSDs. Her myoclonus was treated with clonazepam and it was diminished. The clinical course was compatible with sporadic CJD. Therefore, no other medicines were tried.

\section{Discussion}

In our case, AMT, RMT, and CMCT were normal, while TMS-induced CSP was prominently prolonged. To date, CSP has been measured in only 2 CJD patients [2, 3]. Matsunaga et al. [2] (2000) reported marked prolongation of the CSP in a CJD patient presenting with periodic negative myoclonus associated with PSD on EEG. Ohnari et al. [3] (2006) also reported marked prolongation of the CSP on the affected side in a CJD patient experiencing unilateral periodic positive-negative myoclonus associated with PSD. The common findings in both cases were marked prolongation of CSP, periodic myoclonus, and PSD on EEG. In our case, however, neither periodic myoclonus nor PSD was observed at the time of our TMS experiments. In short, we demonstrated that TMS-induced CSP was prominently prolonged even at the early stage of CJD without periodic myoclonus or PSD.

The CSP was markedly prolonged in all of the studied CJD patients. In our case, the duration of CSP Rмт 120\% $(290 \mathrm{~ms}$ ) equaled that of the mean $+5.2 \mathrm{SD}$ of the normal value, and that of $\mathrm{CSP}_{\text {RMT }} 140 \%$ (370 ms) equaled that of the mean +6.5 SD. Matsunaga et al. [2] reported that the duration of CSP Rмт 110\% was prolonged to $301.7 \mathrm{~ms}$ (+7.2 SD), and Ohnari et al. [3] reported a

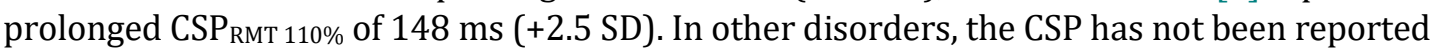
to be comparably prolonged to that of CJD patients. For example, a slight or mild prolongation of the CSP has been reported in patients with stroke $(+1.3 \mathrm{SD})$, multiple system atrophy $(+0.9$ SD), progressive supranuclear palsy (+1.9 SD), and depression (+0.8 SD) [1,5-8]. Moreover, a slight or mild shortening of CSP has been reported in patients with amyotrophic lateral sclerosis, Alzheimer's disease, and Parkinson's disease [1,5]. Considerable shortening of CSP has been demonstrated in patients with corticobasal degeneration (-1.3 SD) [7] and benign adult familial myoclonic epilepsy (-1.3 SD) [2]. As described here, the prominent prolongation of the CSP might be characteristic of CJD.

In summary, we conclude that TMS-induced CSP could be prominently prolonged even in the early stage of CJD. The marked prolongation of the CSP might be an early biomarker of CJD, but further investigations of TMS-induced CSP in a large number of CJD patients would be needed before arriving at a definitive conclusion regarding this issue. 


\section{Case Reports in Neurology}

\section{Statement of Ethics}

The patient and her family provided written informed consent for publication of this paper including images.

\section{Conflict of Interest Statement}

The authors have no disclosures. The authors have no potential conflicts of interest.

\section{Funding Sources}

The authors did not receive any funding related to this paper.

\section{Author Contributions}

Hideyuki Matsumoto wrote the first draft of this paper, which was reviewed by each author. Hideyuki Matsumoto, Naohiro Uchio, and Akihito Hao clinically cared for this patient as neurologists. Hideyuki Matsumoto, Mari Haga, Chiaki Abe, and Yuuri Sakamoto obtained the neurophysiological data. Yoshikazu Ugawa reviewed this paper with critical advice.

\section{References}

1 Cantone M, Di Pino G, Capone F, Piombo M, Chiarello D, Cheeran B, et al. The contribution of transcranial magnetic stimulation in the diagnosis and in the management of dementia. Clin Neurophysiol. 2014 Aug;125(8):1509-32.

2 Matsunaga K, Uozumi T, Akamatsu N, Nagashio Y, Qingrui L, Hashimoto T, et al. Negative myoclonus in Creutzfeldt-Jakob disease. Clin Neurophysiol. 2000 Mar;111(3):471-6.

3 Ohnari K, Matsunaga K, Uozumi T, Tamagawa A, Hashimoto T, Tsuji S. Unilateral positive-negative myoclonus in Creutzfeldt-Jakob disease. Mov Disord. 2006 Nov;21(11):1963-6.

4 Matsumoto H, Saito K, Konoma Y, Okabe S, Ugawa Y, Ishibashi Y. Motor cortical excitability in peritoneal dialysis: a single-pulse TMS study. J Physiol Sci. 2015 Jan;65(1):113-9.

5 Agarwal S, Koch G, Hillis AE, Huynh W, Ward NS, Vucic S, et al. Interrogating cortical function with transcranial magnetic stimulation: insights from neurodegenerative disease and stroke. J Neurol Neurosurg Psychiatry. 2019 Jan;90(1):47-57.

6 Catano A, Houa M, Noël P. Magnetic transcranial stimulation: clinical interest of the silent period in acute and chronic stages of stroke. Electroencephalogr Clin Neurophysiol. 1997 Aug;105(4):290-6.

7 Kühn AA, Grosse P, Holtz K, Brown P, Meyer BU, Kupsch A. Patterns of abnormal motor cortex excitability in atypical parkinsonian syndromes. Clin Neurophysiol. 2004 Aug;115(8):1786-95.

8 Steele JD, Glabus MF, Shajahan PM, Ebmeier KP. Increased cortical inhibition in depression: a prolonged silent period with transcranial magnetic stimulation (TMS). Psychol Med. 2000 May;30(3):565-70. 


\section{Case Reports in Neurology}
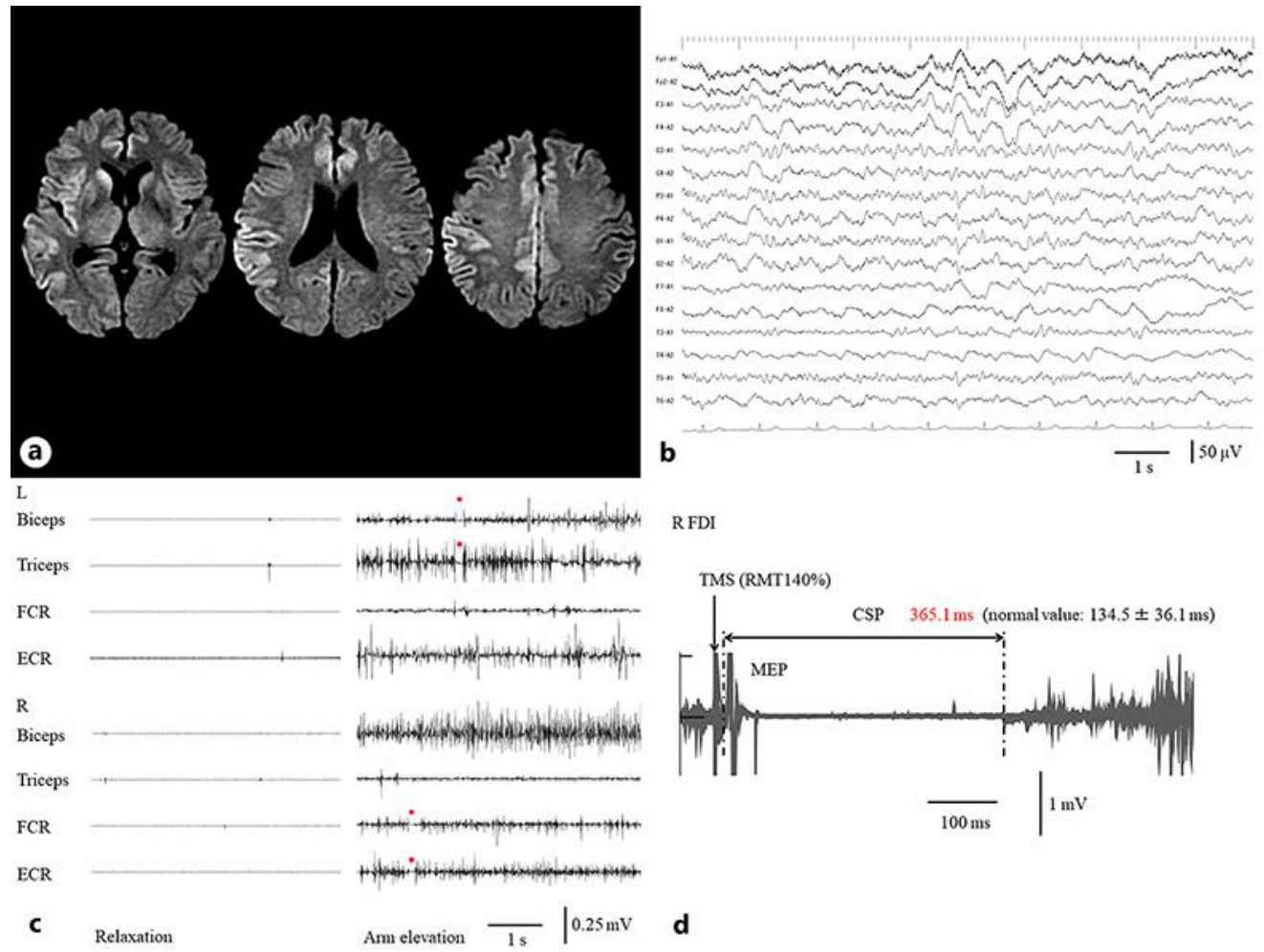

Fig. 1. Neuroradiological and neurophysiological findings in a CJD patient. a Diffusion-weighted MRI shows high-intensity lesions in the cerebral cortex, basal ganglia, and thalamus. b EEG shows diffuse and continuous slow waves, but no PSDs. c Surface EMG shows irregular positive multifocal myoclonus during relaxation, and irregular positive multifocal myoclonus and irregular negative myoclonus during bilateral arm elevation. Red circle indicates negative myoclonus. FCR, flexor carpi radialis; ECR, extensor carpi radialis. d TMS-induced CSP was prominently prolonged. 